\title{
Kernos
}

Revue internationale et pluridisciplinaire de religion grecque antique

19 | 2006

Varia

\section{Michel MALAISE, Pour une terminologie et une analyse des cultes isiaques}

\section{Laurent Bricault}

\section{OpenEdition \\ Journals}

Édition électronique

URL : https://journals.openedition.org/kernos/500

DOI : $10.4000 /$ kernos.500

ISSN : 2034-787

\section{Éditeur}

Centre international d'étude de la religion grecque antique

\section{Édition imprimée}

Date de publication : 1 janvier 2006

Pagination : 490-496

ISSN : 0776-3824

\section{Référence électronique}

Laurent Bricault, "Michel maLAISE, Pour une terminologie et une analyse des cultes isiaques », Kernos

[En ligne], 19| 2006, mis en ligne le 22 mars 2011, consulté le 24 août 2022. URL : http://

journals.openedition.org/kernos/500 ; DOI : https://doi.org/10.4000/kernos.500 
venue expressément révéler aux hommes, c'était rien moins, en effet, qu'un bonheur plénier, illuminé par la connaissance des «principes de la vie », comme dit Cicéron, et mis à l'abri de la crainte de la mort par une promesse eschatologique. Mise à part cette dernière dimension, le rapprochement permet peut-être de mieux comprendre l'attirance, à première vue si étrange, que les cultes éleusiniens ont exercée sur Épicure.

André Motte

(Université de Liège)

Michel MalaIse, Pour une terminologie et une analyse des cultes isiaques, Bruxelles, Académie royale de Belgique, 2005. 1 vol. $16 \times 24,5 \mathrm{~cm}, 282$ p. (Mémoires de la Classe des Lettres-in $8^{\circ}$, $3^{\mathrm{e}}$ série, 35). ISBN : 2-8031-0217-X

Depuis la publication, en 1884, de l'Histoire du culte des divinités d'AlexandrieSérapis, Isis, Harpocrate et Anubis- bors d'Égypte de Georges Lafaye, nos connaissances sur la diffusion du culte de certaines divinités égyptiennes hors de la vallée du Nil se sont diversifiées et approfondies. Le nombre de documents publiés n'a cessé de croître et les études ponctuelles ou plus générales sur le sujet se sont multipliées, engendrant une bibliographie riche aujourd'hui de plus de 8000 titres. Mais tout ceci n'est pas allé sans un certain éparpillement terminologique générateur d'ambiguïtés sinon de confusions, voire d'erreurs d'appréciation, du fait de l'utilisation parfois impropre et souvent indifférenciée qui fut faite des expressions «cultes égyptiens », "cultes nilotiques », "cultes alexandrins » ou " cultes isiaques ». Aussi devenait-il urgent, comme l'écrit M. Malaise (M.M.), «de bien circonscrire l'objet de [ces] études, de clarifier le vocabulaire utilisé, et de bien distinguer les différents types de documents en relation plus ou moins étroite avec l'Égypte ». Ce sont les résultats de cette vaste réflexion méthodologique nourrie par près de quatre décennies de fréquentations isiaques que l'A. nous propose aujourd'hui.

Après avoir rappelé, dans un premier chapitre (p. 15-24), par le biais d'une brève historiographie, les hésitations terminologiques du siècle passé, il analyse (p. 25-31) le sens du mot « isiaque » dans l'Antiquité et chez les modernes pour nous rejoindre sur la définition proposée pour ce terme, en considérant qu'est « isiaque ce qui concerne le culte hors d'Égypte, entre la fin du IV ${ }^{\mathrm{e}}$ s. av. J.-C. et la fin du IV ${ }^{\mathrm{e}}$ s. apr. J.-C., d'une douzaine de divinités appartenant à un même cercle mythique, cultuel et liturgique, originaires de la vallée du Nil, à savoir Anubis/Hermanubis, Apis, Bubastis, Harpocrate, Horus, Hydreios, Isis, Neilos, Nephthys, Osiris et Sarapis. » Les supports de ces croyances, quelle qu'en soit la nature, sont les isiaca. Lorsqu'un doute subsiste sur la valeur cultuelle réelle d'un document, ou s'il s'agit d'une trouvaille isolée, l'A. suggère avec raison de parler simplement d'un «témoignage de type isiaque ».

Le chapitre 3 est l'occasion d'étudier la gens isiaque, c'est-à-dire les divinités du cercle isiaque autres que les membres de la tétrade Isis-Osiris/Sarapis-Anubis-Harpocrate, et d'évaluer leur place, leur(s) rôle(s) et leur importance relative au sein de celleci. Horus (p. 34-41) offre un cas particulièrement complexe, le dieu pouvant revêtir l'aspect d'une divinité solaire et céleste comme celui du fils d'Isis. Pour l'A., il semble bien que dans les textes grecs, les mentions d'Horus nommé seul renvoient la plupart du temps au dieu-faucon, ce qui est certainement exact, tandis que lorsque le théonyme entre dans une séquence isiaque, il désignerait plutôt Harpocrate. Nous ne sommes toujours pas convaincus qu'il en soit systématiquement ainsi et certaines dédicaces macédoniennes paraissent s'adresser à Horus et Harpocrate. On peut d'ailleurs se demander si, à l'instar d'Osiris et de Sarapis, Horus et Harpocrate ne sont pas souvent perçus en milieux hellénisés comme deux entités divines à part entière, dont 
l'une serait antérieure à l'autre, à l'iconographie distincte, pourvues de fonctions différentes, et ce jusqu'à une époque tardive puisqu'au III ${ }^{\mathrm{e}} \mathrm{s}$. apr. J.-C., une liste de prêtres athéniens (L. Bricault, RICIS 101/0216) nomme d'une part un prêtre d'Harpocrate et d'autre part, un peu plus loin, un prêtre d'Horus. Notons en passant que la mention du nom Hôros sur une stèle de Goritza, en Thessalie ne concerne pas le dieu lui-même. Il s'agit en fait d'un nom théophore gravé sur une épitaphe du début du III s. av. J.-C. provenant de la nécropole ${ }^{8}$ à rapprocher du patronyme d'un prêtre d'Isis originaire du Delta, Ouaphrès, fils d'Hôros, dont la stèle funéraire, datable du milieu du III ${ }^{\mathrm{e}} \mathrm{s}$. avant notre ère, fut retrouvée sur le site de l'antique Démétrias, toujours en Thessalie (RICIS 112/0701). Apis (p. 41-51) fut intégré au cercle isiaque pour diverses raisons. Outre les traits communs qu'il partage avec Osiris, il est parfois présenté comme son fils, voire assimilé à Horus. Dans certains cas, il est considéré comme l'époux d'Isis-Hathor. Sa présence, attestée par des documents de caractères bien différents, reste toutefois discrète, d'autant plus que l'identification de certains taureaux en mouvement à des Apis demeure souvent problématique, comme le souligne avec justesse l'A. Il étudie ensuite (p. 51-59) le cas de Boubastis, tantôt identifiée, tantôt associée à Isis, mais aussi parfois indépendante de cette dernière, tout en gravitant dans son orbe en tant que déesse de la maternité. Le théonyme Hydreios, qui n'apparaît pour le moment qu'à Délos (p. 59-66), renvoie à la nature hydriaque d'Osiris, qui se matérialise au moins à partir du principat d'Auguste sous la forme de l'hydrie sacrée. M.M. écrivait en 1972 qu'Hydreios était en quelque sorte une "forme hellénisée d'Osiris »", - ce que nous croyons également -, et l'on peut regretter qu'il n'ait pas développé plus avant son analyse sur ce point dans le présent ouvrage. Ceci d'autant plus que le dossier sur Hydreios s'enrichit désormais d'une étude d'Hélène Siard, qui démontre de façon convaincante que le bâtiment du Sarapieion C de Délos connu jusqu'alors sous le nom de temple C n'est autre que l'Hydreion accueillant Hydreios, c'est-à-dire l'eau salvatrice divinisée. Des fouilles récentes ont en effet révélé la destination hydraulique de ce «temple » dont la cella était occupée tout entière par un grand puits carré, et qui fut édifié à la fin du II $^{\mathrm{e}}$ ou au tout début du $\mathrm{I}^{\mathrm{er}} \mathrm{s}$. av. J.-C. ${ }^{10}$. Au terme de son enquête, elle conclut, contra M.M., qu'Hydreios est une divinité à part entière, représentant la divinisation de l'eau de l'Hydreion, sans référence à Osiris, ce qui nous paraît peu vraisemblable.

La figure de Neilos (p. 66-74), manifestation allégorique du Nil en crue élevée au rang de divinité par les Grecs pose problème, comme le souligne l'A., dans la mesure où son intégration à la famille isiaque ne va pas de soi. Lorsqu'il s'agit en effet d'honorer l'eau de la crue, c'est Hydreios que les isiaques vénèrent, voire l'Osiris dit Canope. La place réelle du Nil est donc délicate à établir, en l'absence de documents probants. Plusieurs textes épigraphiques et littéraires attestent certes des liens unissant Sarapis au Nil, le premier amenant la crue du second, mais nous ne possédons actuellement pas de trace claire d'un culte rendu à Neilos en milieu isiaque.

${ }^{8}$ Cf. G.J.M.J. Te Riele, «An Inscribed Stele », in S.C. Bakhuizen (éd.), A Greek City of the Fourth Century B.C., Roma, 1992, p. 304-305.

${ }^{9}$ M. MALAISE, Les conditions de pénétration et de diffusion des cultes égyptiens en Italie, Leiden, 1972 (EPRO, 22), p. 309.

${ }^{10}$ H. SIARD, «L'hydreion du Sarapieion C de Délos : la divinisation de l'eau dans un sanctuaire isiaque ", in L. BRICAULT et al. (éds), Nile into Tiber. Egypt in the Roman World, 200 BCAD 400, III international congress of Isis studies/III colloque international sur les études isiaques, Leiden University, The Netherlands, May 11-14, 2005, Leiden/Boston, 2006 (RGRW, 159) (à paraitre). 
Tout aussi discrète est la figure de Nephthys, søur cadette d'Isis, en contexte isiaque (p. 74-78). Une épigraphe athénienne l'associant à son frère Osiris, quelques intailles sur lesquelles, accompagnée d'Isis, elle pleure Osiris forment un maigre dossier auquel il faudrait toutefois ajouter plusieurs monnaies de Malte et de Rome, au revers desquelles les deux sœurs, ailées et drapées, entourent la momie du dieu, frappes de la fin du III $^{e}$ ou du début du II $^{\mathrm{e}} \mathrm{s}$. av. J.-C. pour les premières, des dernières décennies du $\mathrm{IV}^{\mathrm{e}} \mathrm{s}$. apr. J.-C. pour les secondes. Il serait intéressant de savoir pour quelle(s) raison(s) fut utilisé, à plus de cinq siècles de distance, si loin de la vallée du Nil, un semblable schéma iconographique. Quoi qu'il en soit, il semble bien qu'hors d'Égypte, la présence de Nephthys ne se conçoive pas en dehors de celle d'Osiris Certains auteurs ont, par le passé, songé retrouver Nephthys sous le théonyme Néôtéra ${ }^{11}$. M.M. montre clairement qu'en réalité cette épiclèse érigée en divinité recouvre des déesses bien différentes, qu'il s'agisse d'Aphrodite(-Hathor), de Déméter, d'une Baalat, de Caelestis, voire, éventuellement, de Nephthys.

D'autres personnages divins, qui ne font pas directement partie de la gens isiaque mais sont parfois mis en relation avec elle, font l'objet du quatrième chapitre. C'est le cas de Bès ${ }^{12}$, protecteur de l'Horus solaire et tuteur d'Harpocrate (p. 80-81). Contra V. Tran tam Tinh, l'A. estime à juste titre que Bès ne fut sans doute pas un sunnaos théos de la famille isiaque, mais qu'il ne faut pas pour autant le négliger, sa présence en un lieu donné pouvant être un indice de l'existence de cultes isiaques sur le site.

Si la figure de Zeus-Ammon-Sarapis apparaît à de nombreuses reprises en Égypte et à Alexandrie ${ }^{13}$, il n'en va pas de même hors de la vallée du Nil et on ne peut guère intégrer Ammon au cercle isiaque (p. 80-85), pas plus d'ailleurs que Thot (p. 85-100) $)^{14}$ et Sobek (p. 100-110), comme le fait ressortir très justement l'A. Notons toutefois que Sarapis et Ammon sont indiscutablement rapprochés sinon identifiés à l'époque impériale en Samarie et en Arabie. Ainsi, au revers d'une monnaie de Néapolis de Samarie au nom de Volusien (251 apr. J.-C.), sont figurés une enseigne rappelant le statut de colonie de vétérans obtenu deux ou trois ans plus tôt, un bélier et un épi de blé qui associent Sarapis à Zeus-Ammon, tandis que le mont Gerizim surplombe la scène ${ }^{15}$. Une autre monnaie, de Bostra cette fois, frappée sous Philippe $\mathrm{I}^{\mathrm{r}}$, présente au revers le buste drapé, cuirassé et diadémé de Sarapis-Ammon, tournée vers la droite, coiffé du calathos, ceint de la taenia et portant sur la tempe la corne de bélier. D'autres

\footnotetext{
${ }^{11}$ Une liste des documents présentant le nom de Néotéra avait été fournie par L. MORETTI, «A proposito di Neotera », Aegyptus 38 (1958), p. 203-209.

${ }^{12}$ Voir déjà M. MALAISE, « Bès et la famille isiaque », $C d E 79$ (2004), p. 266-292.

${ }^{13}$ Il semble en fait que Sarapis-Ammon fasse son entrée dans le monnayage alexandrin dès le règne de Trajan, et non sous Hadrien. Cf. G. DATTARI, A. SAVIO, Catalogo completo della Collezione Dattari, Trieste, 1999 , n 7170 et 12241 .

${ }^{14}$ Une inscription de la fin du II $^{\mathrm{e}}$ ou du début du $\mathrm{III}^{\mathrm{e}}$ s. découverte en 1981 dans le castrum de Statio Cataractarum Dianae, en aval des Portes de fer, s'adresse à un dieu dans lequel V. KONDIC, «Statio Cataractarum Diana », Cabiers des Portes de Fer IV, Belgrade, 1987, p. 45-47, a voulu reconnaître Thot, mettant cette épigraphe en relation avec l'épisode fameux de la «Pluie miraculeuse ». À la suite de M.-Chr. Budischovsky, «Thot aux Portes de fer », in C. Berger et al. (éds), Hommages à Jean Leclant III, Le Caire, 1994, p. 87-95, cela nous paraît fort peu probable. À la bibliographie utilisée par M. Malaise dans sa discussion sur l'histoire de la "Pluie miraculeuse », on ajoutera P. Cordier, «Dion Cassius et les phénomènes religieux "égyptiens". Quelques suggestions pour un mode d'emploi », in L. BriCAUlt et al. (éds), Nile into Tiber (à paraître).

15 A. KINDLER, « Was There A Detachment of The Third Legion Cyrenaica At Neapolis in A.D. 251-253? », INJ 4 (1980), p. 56-58 et pl. 16.6, qui met cette émission en relation avec la présence à Néapolis d'un détachement (vexillatio ou cohors) de la legio III Cyrenaica sous Volusien.
} 
émissions bostréennes légèrement antérieures font probablement apparaître le même dieu dans un temple distyle, s'il faut bien reconnaître un bélier dans l'animal l'accompagnant ${ }^{16}$

Dans la partie conclusive des bonnes pages qu'il consacre aux liens entre Thot/Hermès et la gens isiaque, et notamment Isis, M.M. fait usage du terme isisme, sans doute en référence au riche article consacré par ses soins aux rapports entre la «théologie isiaque » et la gnose ${ }^{17}$, un mot qu'il avait cependant admis ne pas être heureux quelques pages plus haut (p. 26 précisément). Il nous paraît en effet bien difficile de parler d'isisme, d'isiasme ou d'isiacisme, termes que l'on retrouve ici et là, pour qualifier une religion isiaque qui serait cohérente et formerait un tout. De plus, la notion de théologie isiaque (p. 100) mériterait discussion. L'arétalogie d'Isis, d'autres textes, l'initiation justifient-ils la réalité d'une véritable «doctrine isiaque »? Nous n'en sommes pas entièrement convaincu ${ }^{18}$.

S'agissant de Sobek/Souchos, M.M. a sans doute raison de ne pas retrouver dans les représentations du dieu les seules traces d'une quelconque égyptomanie, contrairement à ce que suggèrent les diverses études de Zsolt Kiss sur le sujet. La signification de l'image du saurien n'est d'ailleurs pas univoque. Si le crocodile peut figurer l'Égypte fertile et féconde, il peut aussi connoter la soumission de la terre du Nil, comme sur les monnaies augustéennes à la légende AEGYPTO CAPTA (p. 104), ou sur lesaurei et les sesterces émis par la monnaie de Rome en 215, sur lesquels apparaît au revers Caracalla debout à gauche, en habits militaires, tenant une lance et posant le pied droit sur un crocodile, tandis qu'Isis, debout face à lui, tient un sistre de la main gauche et avance la droite pour présenter à l'Empereur deux épis de blé. Dans cette scène, qui symbolise l'arrivée de l'Empereur en Égypte, le saurien doit représenter les forces mauvaises soumises par Rome, tandis qu'Isis est l'image d'une Égypte prospère et nourricière. Si Sobek fut parfois figuré en liaison avec les divinités du cercle isiaque, c'est en vertu de sa nature de dieu de l'eau, garant de la fertilité des sols. Cependant, aucun document ne permet d'avancer qu'il ait pu bénéficier d'un culte propre hors d'Égypte. Antinoüs, quant à lui, fut honoré en tant que sunthronos du couple isiaque, ainsi que l'attestent deux inscriptions italiennes. Devenu un $\not \mathfrak{s} s y^{19}$, à l'instar d'Osiris, après sa noyade dans le Nil, il fut assimilé au grand dieu égyptien et profita sans doute, durant une courte période, de cet état de fait pour prendre place aux côtés d'Isis et de Sarapis. M.M., qui avait bien noté (p. 51) les liens ayant pu exister entre Apis et Antinoüs, liens suggérés par certaines monnaies micrasiatiques, n’a pas repris ces données dans les pages dédiées au favori d'Hadrien (p. 110-117).

Le chapitre 5 (p. 119-125) est consacré à ce que l'A. appelle avec une grande pertinence la «religion égyptienne isiaque » et les cultes gréco-égyptiens. La première expression se propose de recouvrir sous tous ses aspects le panthéon isiaque en terre d'Égypte durant l'époque gréco-romaine. C'est là un vaste sujet, hors du propos de

${ }^{16}$ Ces monnaies sont étudiées dans la Sylloge Nummorum Religionis Isiacae et Sarapiacae (L. BRICAULT [dir.]), à paraître.

${ }^{17}$ M. MALAISE, «Isisme et gnosticisme », in J. RIES (dir.), Gnosticisme et monde hellénistique. Actes du Colloque de Louvain-la-Neuve 11-14 mars 1980, Louvain-la-Neuve, 1982, p. 47-60.

${ }^{18}$ À propos des logoi contenus dans les livres XXIII-XXVI du Corpus Hermeticum, on peut tirer un grand profit de M. MERTENS, «Une scène d'initiation alchimique : la "lettre d'Isis à Horus" », RHR 205 (1988), p. 3-23.

19 Aux études citées p. 112, on ajoutera G. WAGNER, «Le concept de 'ḥsy' à la lumière des inscriptions grecques », in Egyptian Religion. Studies dedicated to Jan Quaegebeur, Leuven, 1998 (Orientalia Lovaniensia Analecta, 85), vol. II, p. 1073-1078. 
M.M., qui sera abordé par les intervenants du $I V^{*}$ colloque international sur les études isiaques, qui se tiendra à Liège à l'automne 2008. La seconde définit, de manière restrictive mais claire, l'ensemble des cultes rendus aux divinités helléniques qui ont connu une interpretatio aegyptiaca, comme Athéna, Némésis, les Dioscures, Aphrodite et Euthénia, brièvement évoqués dans ces pages.

Le chapitre suivant, beaucoup plus dense (p. 127-180), s'attache aux « cultes alexandrins », c'est-à-dire ceux de «divinités égypto-grecques qui seraient non seulement le résultat d'une interpretatio graeca d'anciennes divinités égyptiennes, mais, qui, en outre, offriraient des traits spécifiques à la grande ville portuaire. » (p. 128). Le premier d'entre eux, Sarapis, est présenté en une dizaine de pages qui ont le grand intérêt de faire commodément ressortir les enjeux principaux de la personnalité et du culte du dieu $^{20}$, dont l'A. admet finalement, au terme de deux excellentes pages (p. 138-139), qu'il est «avant tout alexandrin dans la mesure où sa nouvelle iconographie fut inventée dans la ville d'Alexandre ». Pour le reste, il est clair qu'il faut chercher ailleurs, à Memphis sans doute, les origines du dieu. Deux questions, essentielles nous semble-til, ne sont toutefois pas traitées par l'A.. Quand précisément Isis devint-elle l'épouse de Sarapis, ce que nombre de textes alexandrins attestent dès le milieu voire la fin du premier quart du III $^{\mathrm{e}} \mathrm{s}$. av. J.-C., comme M.M. le rappelle p. 152, et cette hiérogamie fut-elle célébrée à Alexandrie même ? Sarapis fut-il dès l'origine un dieu guérisseur et oraculaire? Osiris, de son côté, est quasiment absent d'Alexandrie, sauf sous son aspect proprement hydriaque, comme l'attestent les monnaies de la cité et une statue repêchée au large (p. 139-141). Isis, dont le culte est attesté dans la ville par un nombre de documents finalement moins important qu'on ne pourrait le supposer (p. 141-152), a-t-elle été adorée à Alexandrie sous une forme particulière? M.M. répond par la négative, à juste titre. Ni Isis Pharia, ni Isis Lochia ${ }^{21}$, ni Isis lactans ne peuvent être considérées comme de véritables Isis alexandrines. Il en va de même pour Harpocrate (p. 152-154), même si le modèle praxitélien du dieu fut peut-être élaboré dans la capitale des Ptolémées, ce qui reste d'ailleurs à démontrer, ainsi que pour Anubis (p. 154-157). En revanche, la figure d'Euthénia pourrait bien être spécifique d'Alexandrie, si l'on en croit l'immense majorité des documents la représentant (p. 157-158). En alla-t-il de même pour Agathos Daimôn, comme on l'a très souvent écrit jusqu'à présent? La question, délicate tant les sources le concernant sont peu explicites et leur interprétation parfois suspecte, est abondamment discutée par l'A. (p. 158-176). Pour lui, il est vraisemblable que le Bon Génie d'Alexandrie partagea avec Sarapis, sans doute dès l'époque ptolémaïque, le rôle de divinité tutélaire de la ville, veillant sur les destinées de la capitale des Ptolémées, que l'on trouve à l'occasion personnifiée, comme sur de très nombreuses monnaies d'époque impériale (p. 176-177), tenant à l'occasion une tête de Sarapis dans sa paume droite tendue.

Dans le court chapitre suivant (p. 181-191), M.M. s'interroge sur la portée de ces diverses interpretationes, au travers de quelques exemples, ceux d'Hermanubis, d'Héliosérapis ${ }^{22}$ et d'Harpocrate, constatant que les images isiaques, les représentations

\footnotetext{
${ }^{20}$ Il revient sur la « création » de Sarapis plus loin, p. 164-166.

${ }^{21}$ Il est très probable, ainsi que le suggère M. Malaise (p. 150, n. 160), que l'épiclèse portée à Bouto par Isis d'après le $P$. Oxy. XI, 1380, (1. 27) soit lo[chian]. Dans notre thèse demeurée inédite, nous proposions de lire lo[chian, Esenchébin].

${ }^{22}$ Sur cet aspect syncrétique, et les problèmes de correspondance entre un théonyme et l'image qui pourrait le matérialiser iconographiquement, $c f$. récemment L. BRICAULT, «Zeus Hélios mégas Sarapis ", in C. CANNUYER et al. (éds), La langue dans tous ses états. Michel Malaise in bonorem, Louvain-la-Neuve, 2005 (Acta Orientalia Belgica, 18), p. 243-254.
} 
gréco-égyptiennes, les théonymes étaient parfois interchangeables, et que diverses formes de «syncrétisme » unissant les uns et les autres pouvaient être déterminées. Ces quelques pages, et celles du chapitre suivant intitulé « polymorphie et polysémie » (p. 193-199) auraient mérité d'être développées, tant le phénomène est complexe et au cour de bien des débats. Une grille de lecture des différents processus syncrétiques mis en ouvre au sein des cultes isiaques n'aurait pas été inutile. Sans doute même, dans cet essai méthodologique, l'A. aurait-il pu, avec grand profit, aller jusqu'à poser le problème crucial de la dénomination des images divines, et poser les critères permettant de les définir, de les nommer. Les images comme les mots sont polysémiques.

Un dernier chapitre, le neuvième (p. 201-220), propose de définir les termes $A e$ gyptiaca, Pharaonica, Nilotica et Aegyptomania. À la suite de J. Yoyotte, M.M. propose de considérer comme aegyptiaca «les trouvailles faites hors d'Égypte et du Soudan de produits authentiquement égyptiens et d'imitations peu ou prou égyptisantes » (p. 202), sans la restriction chronologique que nous avions proposée ${ }^{23}$, et que nous abandonnons volontiers. Lorsque ces aegyptiaca sont intégrés à un contexte isiaque, on parlerait de pharaonica ${ }^{24}$. Ces définitions sont loin d'être acceptées par tous $^{25}$, et forment le sujet d'un profond débat, qui dépasse largement le cadre de simples étiquettes lexicales. On peut d'ailleurs se demander ce que recouvre précisément le terme "égyptisant», tant son acception semble varier d'un auteur à l'autre. Les nilotica désigneraient les scènes montrant le Nil en crue et la campagne égyptienne ainsi fertilisée, représentations sur des supports très divers et dont la signification précise varie d'un lieu à un autre, d'un propriétaire à l'autre. Généralement étrangères aux contextes isiaques, de telles scènes leurs sont cependant parfois associées. Quant à l'Aegyptomania, réservée au domaine artistique, elle serait « un phénomène complexe qui mêle des éléments néo-égyptiens empruntés à l'art de l'Égypte antique puis réinterprétés et réemployés, et des éléments néo-égyptisants issus d'une égyptomanie antérieure », selon les mots de J.-M. Humbert ${ }^{26}$, que l'A. reprend à son compte (p. 214). Les traces les plus anciennes d'égyptomanie se trouveraient alors dans l'Italie des $\mathrm{II}^{\mathrm{e}}-\mathrm{I}^{\text {er }}$ S. av. J.-C. Pour autant, peut-on considérer les marques monétaires présentant des types qualifiés d'égyptiens (basileion, sistre, situle, fleur de lotus, chameau, crocodile, ibis, ichneumon, pygmée, rhinocéros, serpent) comme les marques d'une « égyptomanie ambiante » (p. 220) ? Sans doute pas. Mais M.M. a raison de faire justice de toutes les théories qui, d'E.A. Sydenham à F. Coarelli, en passant par A. Alföldi ont voulu attribuer à ces quelques marques monétaires une importance pour le moins usurpée. Il est clair que l'utilisation particulièrement sporadique de motifs réellement isiaques, par une huitaine de monétaires ${ }^{27}$ dont aucun, a priori, ne peut être suspecté d'affinités avec le culte de la déesse ou ses dévots ${ }^{28}$, invite à ne pas attribuer à ces

${ }^{23}$ L. BRICAUlT, «Études isiaques : bilan et perspectives », in E. Leospo, D. Taverna (éds), $L a$ Grande Dea tra passato e presente. Tropi Isiaci I, Torino, 2000, p. 92.

${ }^{24}$ Parmi lesquels certaines statues naophores étudiées par M. MALAISE, «Statues égyptiennes naophores et cultes isiaques », BSEG 26 (2005), p. 63-80.

${ }^{25}$ Cf., par exemple, M.J. Versluys, Aegyptiaca Romana. Nilotic Scenes and the Roman Views of Egypt, Leiden/Boston, 2002 ( $R G R W, 144)$, et la recension en forme de chronique de M. MALAISE, « La signification des scènes nilotiques dans la culture romaine », CdE 78 (2003), p. 308-325.

${ }^{26}$ J.-M. HumberT, L'Égypte à Paris, Paris, 1988, p. 25.

${ }^{27}$ P. 219, il s'agit de C. Calpurnius Piso Frugi, et non Frigonis.

${ }^{28}$ En l'état actuel, très parcellaire, de nos connaissances. On ne peut toutefois écarter complètement cette idée et nous partageons l'opinion mesurée de S. TAKACS, Isis and Sarapis in the Roman World, Leiden, 1995 (RGRW, 124), p. 39, lorsqu'elle écrit que «the significance of these 
images de signification commune, encore moins à les parer d'un message révolutionnaire. Ceci étant, le simple fait de les avoir utilisés au milieu de dizaines d'autres motifs montre qu'ils étaient devenus familiers aux Romains du I ${ }^{\text {er }}$ s. av. J.-C., comme le basileion le fut un siècle plus tôt dans les cités du pourtour égéen.

En guise de conclusion, l'A. fait la synthèse de ses principales prises de position. Une riche bibliographie de presque trente pages, à laquelle il n'y a guère à ajouter, et un bon index d'une vingtaine de pages, analytique et clair, complètent le volume.

Dans cet ouvrage écrit avec le souci de précision qui caractérise l'A. depuis ses premières études, on ne relève que peu de coquilles. Rappelons simplement que p. 76, le prénom de notre collègue Egelhaaf-Gaiser est Ulrike et non Ursula, et celui de S. Handler (p. 142 n. 94, p. 152, p. 163 n. 230) comme de S. Walker (p. 161 n. 214) Susan et non Suzan.

Tel qu'il se présente, l'essai méthodologique que nous propose M.M. marque une étape décisive dans l'histoire des études isiaques. Sa consultation sera indispensable pour permettre à tous ceux, spécialistes ou non, qui se retrouveront confrontés à un document afférent à ce champ de recherche, de distinguer de quoi il retourne pour l'analyser en connaissance de cause. On ne peut qu'en remercier l'A., et attendre avec impatience sa prochaine étude, qui s'attachera à identifier le milieu dans lequel se sont fondus les éléments égyptiens et grecs qui donnèrent par la suite naissance à ce que nous appelons désormais les cultes isiaques.

Laurent Bricault

(Poitiers)

Simone Follet (éd.), L'Hellénisme d'époque romaine. Nouveaux documents, nouvelles approches ( $I^{e r}$ s. a.C. - III s. p.C.). Actes du Colloque international à la mémoire de Louis Robert. Paris, 7-8 juillet 2000, Paris, De Boccard, 2004. 1 vol. $16 \times 24$ cm, 452 p., 34 fig. (De l'Archéologie à l'Histoire). ISBN : 2-7018$0174-5$.

Le présent volume rassemble, sous forme d'un hommage au maître incontesté des études épigraphiques que fut en France Louis Robert, les communications présentées lors d'un colloque organisé par l'Année épigraphique et l'Université de Paris IVSorbonne, autour d'un thème volontairement général, «L'hellénisme d'époque romaine ", mais propice à la mise en valeur de documents nouveaux ou peu étudiés permettant de renouveler notre approche de l'histoire du monde grec à l'époque romaine, c'est-à-dire dans un champ chronologique allant du II $^{\mathrm{e}} \mathrm{s}$. av. J.-C au $\mathrm{III}^{\mathrm{e}} \mathrm{s}$. de notre ère.

La matière des 23 contributions présentées ici a été organisée selon un ordre géographique. Un premier groupe concerne la Macédoine et la Grèce propre (en dehors d'Athènes), très nettement en retrait dans ce colloque, puisque, si l'on excepte quelques références à des cités de la province d'Achaïe dans la communication d'Athanase Rizakis sur l'intervention des empereurs dans la vie économique des cités, une seule communication, celle d'Élodie Cairon sur quatre épigrammes funéraires d'Argos à l'époque hellénistique, porte sur une cité de Grèce propre. Les autres proposent, pour la Macédoine, un examen de dédicaces publiques du I ${ }^{\text {er }}$ S. av. ou ap. J.-C. restées en place au fil des siècles sur le forum de Philippes (Michel Sève) et une étude de ce que les inscriptions du sanctuaire de Leukopétra révèlent de l'influence romaine dans les

pictograms was the outlet they provided for the expression of individuality and, perhaps, personal interest ». 